\title{
Superfícies protetoras na diminuição de danos mecânicos em tomate de mesa
}

\author{
Protective surfaces for reduction of mechanical damages on fresh market tomatoes
}

\author{
Ana Maria de Magalhães ${ }^{\mathrm{I}}$ Marcos David Ferreira ${ }^{\mathrm{I} *}$ Oscar Antonio Braunbeck ${ }^{\mathrm{II}}$ \\ Marcos Vênicius Ramos Estevom ${ }^{\text {III }}$
}

\begin{abstract}
A perda de qualidade do tomate está relacionada RESUMO muitas vezes a danos mecânicos que ocorrem desde a colheita
até o consumidor, quando os frutos são submetidos a quedas em superfícies não-protegidas ou colidem com outros frutos. O objetivo deste trabalho foi avaliar diferentes superfícies $e$ três alturas de queda com relação à magnitude de impacto e à influência na qualidade do tomate de mesa. Para mensurar a magnitude de impacto, utilizou-se a esfera instrumentada. Já a qualidade dos frutos foi avaliada por meio da análise dos danos internos. Para isso, utilizaram-se três superfícies que apresentaram níveis diferentes de impacto combinadas às três alturas de queda. Como resultado, observou-se que as superfícies emborrachadas, por apresentarem uma maior elasticidade, combinadas a menores alturas de queda, demonstram uma maior absorção do impacto $e$ conseqüentemente menor incidência de danos mecânicos.
\end{abstract}

Palavras-chave: Lycopersicon esculentum Mill., altura de queda, qualidade.

\section{ABSTRACT}

Quality losses of fresh market fruit are often related to mechanical damages that occur from harvest thru final consumer. Those damages normally occur when fruits drop from high heights or collide against not protected surfaces or against other fruits. The main goal of this work was to evaluate in laboratory for impact magnitude and tomato quality different surfaces in three drop heights. For measuring impact magnitude it was used the instrumented sphere and for tomato quality the evaluation was based on internal bruising. Fruit drop tests were done using three protective surfaces that showed different levels of maximum acceleration combined with three different heights. It was concluded that the use of determined protective surfaces rubber plate, under low drop high can decrease internal bruising incidence and impact magnitude on packing lines.

Key words: Lycopersicon esculentum Mill., drop height, quality.

As principais causas dos danos mecânicos em produtos hortícolas são provenientes de forças externas de vibração, compressão e impacto, que podem causar diferentes tipos de lesões, como abrasões, cortes, rupturas e danos internos (SARGENT et al., 1989). Em geral, danos por impacto na pós-colheita podem ocorrer quando os frutos sofrem quedas de alturas elevadas ou colidem com superfícies nãoprotegidas. Em uma linha de classificação, esses danos podem ser reduzidos diminuindo a altura de queda ou através da utilização de protetores, os quais podem dissipar a força de impacto (HYDE \& ZHANG, 1992).

Durante o impacto, desenvolvem-se pressões nas áreas de contato que geram tensões de maior intensidade nos tecidos vizinhos, mas que se propagam a toda a massa de tecidos, provocando acelerações. Embora essas acelerações variem em diferentes regiões do fruto, um valor médio representativo da magnitude das mesmas pode ser obtido utilizando uma esfera instrumentada, que é um equipamento plástico dotado de acelerômetros e recursos para registro de dados no tempo. Os níveis de impactos são medidos em termos de aceleração

ITecnologia Pós-colheita, Faculdade de Engenharia Agrícola (FEAGRI), Universidade de Campinas (UNICAMP), Cidade Universitária Zeferino Vaz, CP 6011, 13083-875, Campinas, SP, Brasil. E-mail: marcos.ferreira@agr.unicamp.br. *Autor para correspondência. ${ }^{\mathrm{II} M a ́ q u i n a s ~ A g r i ́ c o l a s, ~ F E A G R I, ~ U N I C A M P, ~ C a m p i n a s, ~ S P, ~ B r a s i l . ~}$

IIILaboratório Unidade Móvel de Auxílio à Colheita (UNIMAC), FEAGRI, UNICAMP, Campinas, SP, Brasil. 
máxima (G, $\left.\mathrm{m} \mathrm{s}^{-2}\right)$ e mudanças na velocidade $\left(\Delta \mathrm{v}, \mathrm{m} \mathrm{s}^{-1}\right)$. À medida que se aumenta a altura de queda, maior será a aceleração máxima e a mudança de velocidade (SARGENT et al., 1992). Além do valor de aceleração descrito, pode ser obtido um valor médio representativo da força desenvolvida na área de contato. Esta força surge como conseqüência das deformações impostas ao material da superfície durante o impacto. A energia cinética dos materiais que colidem transforma-se em energia de deformação dos mesmos. Conhecida a energia cinética dos corpos e o comportamento mecânico do material, pode-se estimar a força máxima de impacto.

O estudo foi realizado em três etapas distintas. No primeiro ensaio, utilizou-se a esfera instrumentada de diâmetro 70mm (Techmark, Inc., Lansing, EUA), que foi lançada através de um equipamento de sucção (Figura 1) desenvolvido por CAMARGO et al. (2004) a partir de três diferentes alturas: 10, 20 e $30 \mathrm{~cm}$, em oito diferentes superfícies protetoras: (1) placa de borracha esponjosa 9,5mm; (2) placa de borracha esponjosa 6,5mm; (3) placa de E.V.A. (Etil Vinil Acetato) 3mm; (4) courvim acoplado a espuma 8mm; (5) lençol de borracha natural 5,5mm; (6) lençol de borracha natural 1,5mm; (7) bagun 0,5mm; e (8) placa de aço carbono $5 \mathrm{~mm}$. Após o ensaio, os dados foram transferidos para o microcomputador e, através das médias da aceleração máxima, determinou-se a magnitude do impacto. Na segunda etapa, foi estimada a força máxima desenvolvida durante o impacto da esfera instrumentada com cada superfície e para cada altura. Para isso, utilizou-se uma esfera de borracha, com o mesmo diâmetro e peso da esfera instrumentada e com aproximadamente a mesma rigidez, que foi apoiada sobre cada um dos materiais em estudo e submetida à compressão em prensa de pratos planos e paralelos, com velocidade de deslocamento de $0,4 \mathrm{~mm} \mathrm{~s}^{-1}$ (Texture Measuring System Canners Machinery Limited Simcol, Canada, modelo D18040, com carga máxima de 450kg). Durante o ensaio, foram registradas a força (N) e o tempo (s) utilizando-se o software SPIRE. Com os resultados, integrou-se a curva força $(\mathrm{N})$ por deformação (mm), cuja área representa a energia $\left(\mathrm{N} \mathrm{mm}^{-1}\right)$ gasta para deformar o material de cobertura. Como a energia cinética acumulada na esfera no início do impacto é conhecida e considerando-se que não foi observado ricocheteamento durante o impacto, podese considerar que a energia cinética da esfera transforma-se integralmente em energia de deformação do material de cobertura. Como a rigidez da esfera é muito superior à da superfície, foi considerado que a energia de deformação corresponde apenas à superfície. Para determinar a força máxima atingida durante o impacto da esfera sobre cada superfície, igualou-se a energia de deformação com a energia cinética de queda da esfera instrumentada (mgh), onde m é a massa da esfera $(0,162 \mathrm{~kg})$, g é a aceleração da gravidade $\left(9,8 \mathrm{~m} \cdot \mathrm{s}^{-2}\right)$ e h é a altura de queda (10, 20 e $30 \mathrm{~cm})$. Devido à estrutura do equipamento, este ensaio não foi realizado para a superfície de aço carbono, pois a alta rigidez deste material não permitiria registrar deformações significativas e poderia danificar o equipamento. Através destes resultados, foi possível conhecer a força máxima desenvolvida em cada caso, visando a utilizar esta informação como parâmetro de estudo, já que se espera que maiores forças máximas estejam associadas com maiores danos aos tecidos do produto.

Na terceira etapa, frutos de tomate "Débora”, no estádio de maturação verde-maduro, adquiridos na CEASA Campinas, foram submetidos à queda por meio do equipamento de sucção. Para este ensaio, utilizaramse três alturas de quedas $(10,20$ e $30 \mathrm{~cm})$ e três superfícies, determinadas através dos resultados obtidos com a esfera instrumentada, que apresentaram valores de aceleração média: baixos = borracha esponjosa 9,5mm, intermediários $=$ EVA. $3 \mathrm{~mm}$ e altos $=$ aço carbono $5 \mathrm{~mm}$. Em cada uma das superfícies, foi aplicado pó de giz para que o fruto ficasse marcado na hora da queda, possibilitando determinar onde ocorreu o dano. Após o ensaio, os frutos foram armazenados em temperatura ambiente $\left(24^{\circ} \mathrm{C}\right)$ juntamente com os frutos referência. Passados sete dias de armazenamento, avaliou-se a presença de danos internos de impacto. Para isso, cada tomate foi cortado transversalmente à marcação e avaliado usando a seguinte escala: 1=nenhum dano físico; 2=mínima descoloração do tecido locular; 3=descoloração do pericarpo e deformação do tecido locular; 4=tecido locular separado dos demais (SARGENT et al., 1992).

Os ensaios foram planejados a partir de Delineamento Inteiramente Casualizado (DIC) em esquema fatorial. Os fatores considerados no ensaio com a esfera instrumentada foram três alturas de queda e oito superfícies, com cinco repetições. No ensaio com os tomates, utilizaram-se vinte frutos para cada tratamento (três alturas de queda e três superfícies, totalizando nove tratamentos). Os resultados foram avaliados por meio da análise de variância e, para a diferença entre as médias, foi aplicado o teste de Tukey a $5 \%$ de probabilidade.

A superfície borracha esponjosa 9,5mm apresentou a melhor absorção do impacto, apresentando uma redução na aceleração em 58, 40 e $38 \%$, quando comparada à superfície aço carbono, para as alturas de queda 10, 20 e $30 \mathrm{~cm}$, respectivamente. A superfície EVA 3mm apresentou valores intermediários de aceleração máxima, apresentando uma redução no impacto de 33, 14 e 13\%, respectivamente, também quando comparada à superfície de aço carbono, para as mesmas alturas de queda. TIMM \& BROWN (1991) também relatam uma diminuição no impacto em linhas de classificação de abacate, mamão e abacaxi, quando 


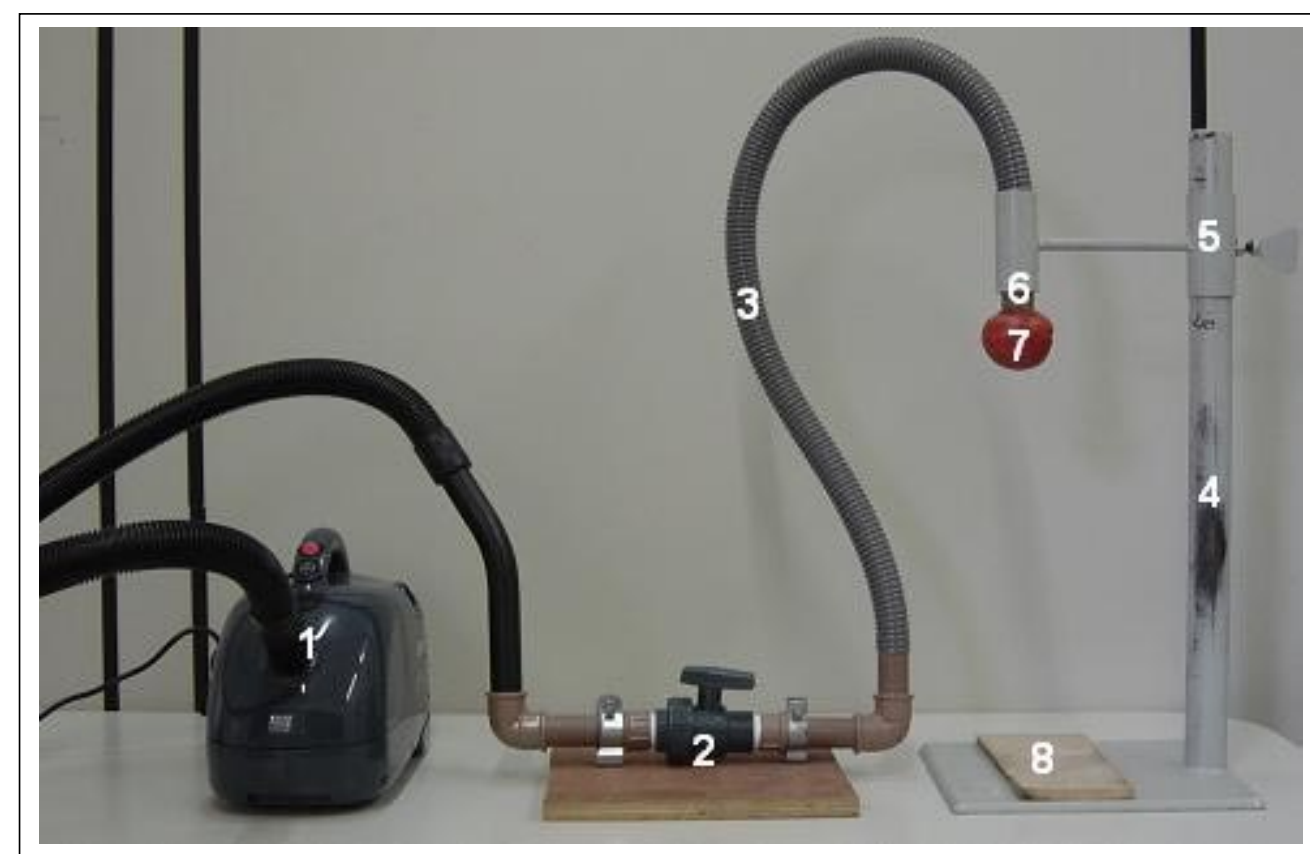

Figura 1- Equipamento desenvolvido para proporcionar queda livre de frutos em laboratório. 1) aspirador de pó portátil com 1000 watts de potência; 2) registro de esfera PVC de 1 polegada; 3) mangueira de plástico, diâmetro $34 \mathrm{~mm}$; 4) suporte de lançamento de chapa de aço carbono, com $60 \mathrm{~cm}$ de altura; 5) ajuste regulador da altura de queda; 6) bocal para segurar os frutos, constituído por uma ventosa de plástico com uma incisão circular no centro para a passagem do vácuo; 7) fruto de tomate; 8) superfície de borracha esponjosa 9,5mm.

da utilização de protetores emborrachados. A superfície aço carbono apresentou as maiores médias para a aceleração máxima nas alturas de 10 e $30 \mathrm{~cm}, 155$ e $280 \mathrm{~m}$ $\mathrm{s}^{-2}$, respectivamente. Na altura de $20 \mathrm{~cm}$, o maior impacto foi observado na superfície lençol de borracha $1,5 \mathrm{~mm}$, com uma aceleração máxima de $220 \mathrm{~m} \mathrm{~s}^{-2}$ (Tabela 1). LOPRESTI \& THOMSON (1998) demonstraram que $40 \%$ dos piores impactos em uma linha de classificação e embalagem de batata consistiram em quedas em superfícies não-protegidas ou finamente protegidas. MILLER \& WAGNER (1991) relatam que 80\% dos impactos descritos em uma linha de classificação para citrus variam entre a faixa de 25-150G e são causados por quedas em superfícies rígidas de metal ou em uma camada de cobertura emborrachada muito fina.

De um modo geral, as superfícies que apresentaram maior absorção de impacto (G, m s-2) demonstraram menor força $(\mathrm{N})$ no ensaio de compressão. Esta força também foi diretamente proporcional com o aumento da altura de queda, dobrando o valor da força para praticamente todas as superfícies, quando se comparou as alturas de 10 e $30 \mathrm{~cm}$ (Tabela 1). Os valores de força obtidos no ensaio com a superfície borracha esponjosa 9,5mm foram os menores encontrados (19,02; 29,81 e 40,75N) para as três alturas de queda (10, 20 e $30 \mathrm{~cm}$, respectivamente), o que demonstrou que durante a queda da esfera nesta superfície não foram aplicados grandes valores de força para a deformação, mostrando que esta superfície apresenta uma estrutura mais flexível do que as demais; portanto, com uma maior absorção do impacto. Todavia, as superfícies bagun $0,5 \mathrm{~mm}$, lençol de borracha natural 1,5mm e lençol de borracha natural 5,5mm apresentaram os maiores valores $(35,43 ; 53,79$ e $68,5 \mathrm{~N})$ para as alturas de 10,20 e $30 \mathrm{~cm}$, respectivamente, ou seja, foram necessários maiores valores de força para deformar essas superfícies. Observa-se, portanto, que, para a redução do impacto, a superfície protetora deverá possuir algum outro tipo de atributo, que forneça elasticidade ao material e, em conseqüência, atue na absorção do impacto de queda do produto. Visando à redução de custos, muitas vezes, em equipamentos de beneficiamento e classificação, utiliza-se somente uma lona plástica nos pontos de transferência de produtos, a qual, conforme demonstram os resultados obtidos, não possui função de absorção de impacto.

Através dos resultados obtidos com a esfera instrumentada, determinaram-se as superfícies a serem utilizadas nos ensaios com os frutos, ou seja, borracha esponjosa 9,5mm, E.V.A. 3mm e aço carbono, que apresentaram valores baixos (60 a $\left.175 \mathrm{~m} \mathrm{~s}^{-2}\right)$, intermediários (100 a 250 $\mathrm{m} \mathrm{s}^{-2}$ ) e altos (150 a 280 m s $\mathrm{m}^{-2}$ ) de aceleração máxima, respectivamente.

Ciência Rural, v.37, n.3, mai-jun, 2007. 
Tabela 1 - Valores da aceleração máxima encontrados utilizando-se a esfera instrumentada (70mm) e da força máxima obtidos no ensaio de compressão, para cada superfície e altura de queda.

\begin{tabular}{|c|c|c|c|c|c|c|}
\hline \multirow{3}{*}{ Superfícies } & \multicolumn{3}{|c|}{ Aceleração máxima [G; m s²] } & \multicolumn{3}{|c|}{ Força máxima [N] } \\
\hline & \multicolumn{6}{|c|}{ Altura de queda [cm] } \\
\hline & 10 & 20 & 30 & 10 & 20 & 30 \\
\hline (1) & 64,40 & 124,42 & 173,10 & 19,02 & 29,81 & 40,75 \\
\hline (2) & 80,04 & 147,72 & 198,37 & 20,80 & 33,07 & 43,42 \\
\hline (3) & 103,68 & 178,77 & 244,61 & 27,30 & 42,23 & 54,23 \\
\hline (4) & 115,27 & 193,13 & 253,34 & 20,80 & 38,68 & 54,38 \\
\hline (5) & 135,55 & 190,34 & 267,14 & 34,84 & 52,60 & 68,50 \\
\hline (6) & 148,16 & 219,60 & 272,30 & 34,55 & 53,79 & 64,04 \\
\hline (7) & 135,46 & 217,91 & 274,60 & 35,43 & 53,49 & 63,15 \\
\hline (8) & 154,34 & 208,51 & 280,35 & --- & --- & ---- \\
\hline
\end{tabular}

Superfícies - (1) borracha esponjosa 9,5mm; (2) borracha esponjosa 6,5mm; (3) Etil Vinil Acetato (EVA) 3mm; (4) courvim acoplado a espuma 8mm; (5) lençol de borracha natural 5,5mm; (6) lençol de borracha natural 1,5mm; (7) bagun 0,5mm; e (8) aço carbono 5mm.

Com o aumento da altura de queda, observou-se um incremento na incidência dos danos internos dos tomates, para todos os tratamentos, assim como foi observado um aumento na magnitude do impacto ( $\mathrm{G} ; \mathrm{m} \mathrm{s}^{-2}$ ) e incremento nos valores de força $(\mathrm{N})$ para as maiores alturas (Tabela 1 ). CAMARGO et al. (2004) e SARGENT et al. (1992) também observaram esta mesma situação em ensaios com tomate de mesa. A superfície borracha esponjosa $9,5 \mathrm{~mm}$ proporcionou uma redução de aproximadamente $20 \%$ nos valores obtidos para o dano interno, quando comparada à superfície aço carbono, e uma redução média de $18 \%$ nas injúrias internas foram observadas na superfície EVA 3mm, quando comparada àquela mesma superfície. Observa-se, portanto, que a superfície borracha esponjosa $9,5 \mathrm{~mm}$, por ser um material de maior elasticidade, absorveu uma parte do impacto de queda do tomate e causou menores danos internos aos frutos. Para SARGENT et al. (1992), modificações em alguns pontos da linha de classificação de tomates de mesa, através da utilização de menores pontos de queda e de protetores de superfície, proporcionaram redução em até $50 \%$ na pressão de impacto, com conseqüente prevenção de danos físicos.

As superfícies emborrachadas, por apresentarem uma maior elasticidade, absorvem melhor o impacto e, em conseqüência, proporcionam menor incidência de danos mecânicos. Os dois métodos utilizados para caracterizar as superfícies, utilizando-se a esfera instrumentada, ou através do teste de compressão, demonstraram serem complementares, podendo ser aplicados, no futuro, para outras superfícies e texturas.

\section{AGRADECIMENTOS}

À Coordenação de Aperfeiçoamento de Pessoal de Nível Superior (CAPES), pela concessão de bolsa de mestrado para o primeiro autor. À Fundação de Amparo à Pesquisa do Estado de São Paulo (FAPESP), pelo financiamento do projeto UNIMAC* - Unidade Móvel de Auxílio à Colheita.

\section{REFERÊNCIAS}

CAMARGO, G.G.R. et al. Testes de impacto em laboratório para tomate de mesa (Lycopersicon esculentum Mill.) cultivar Débora. Horticultura Brasileira, v.22, n.2, p.????, 2004.

HYDE, G.M.; ZHANG, W. Apple bruising research update: packingline impact evaluations. Tree Fruit Postharvest Journal, v.3, n.3, p.12-15, 1992.

LOPRESTI, J.; THOMSON, G. Instrumented sphere assessment of seed potato packing lines. ExpHORT 2000 Report. 1p. Victoria, Australia: Institute for Horticultural Development Knoxfield, 1998. Acesso: 29 jan. 2003. Online. Disponível em: "http://www.nre.vic.gov.au/agvic/ihd/r\&d/doc095.htm\#pagetop>.

MILLER, W.M.; WAGNER, C. Florida citrus packing line studies with an instrumented sphere. Applied Engineering in Agriculture, v.7, n.5, p.577-581, 1991.

SARGENT, S.A. et al. Assessment of mechanical damage in tomato packing lines. Transactions of the ASAE, v.30, n.1, p.630-634, 1989.

SARGENT, S.A. et al. Sensitivity of tomatoes at mature-green and breaker ripeness stages to internal bruising. Journal of the American Society for Horticultural Science, v.117, n.1, p.119-123, 1992.

TIMM, E.J.; BROWN, G.K. Impacts recorded on avocado, papaya, and pineapple packing lines. Applied Engineering in Agriculture, v.7, n.4, p.418-422, 1991. 